\title{
The Association between Changes in Low Parathyroid Hormone Levels and Cardiac Function Decline in Maintenance Hemodialysis Patients: A Prospective Observational Study
}

\author{
Xiao-han Lu, ${ }^{\mathrm{a}}$ beng-si $\mathrm{Li}^{\mathrm{a}}$ Yao-yao $\mathrm{Li}^{\mathrm{a}}$ Yan-dan Zheng ${ }^{\mathrm{a}}$ Xiao-yan $\mathrm{Wu}^{\mathrm{a}}$ \\ Ping $\mathrm{GaO}^{\mathrm{a}}$ \\ aDepartment of Nephrology, Zhong Nan Hospital of Wuhan University, Wuhan, China; ${ }^{\mathrm{b}}$ Department of Nephrology, \\ Xiangyang Central Hospital, Affiliated Hospital of Hubei University of Arts and Science, Xiangyang, China
}

\section{Highlights of the Study}

- Persistently low PTH levels are associated with cardiac function decline in MHD patients.

- The variability in a low PTH serum status is a strong risk factor for cardiac function decline.

- The presence of low PTH levels should be given attention in clinical work.

\section{Keywords}

Cardiology · Renal disease · Maintenance hemodialysis ·

Parathyroid hormone $\cdot$ Cardiac function

\begin{abstract}
Objectives: The aim of this study was to investigate a possible association between changes in low parathyroid hormone (PTH) levels and cardiac function decline in maintenance hemodialysis (MHD) patients. Methods: A total of 150 MHD patients were included and followed for 24 months. The enrolled patients were divided into 3 groups based on their PTH status at baseline and 24 months. Factors potentially involved in changes in the PTH level and cardiac function were compared using multivariate logistic regression analysis. Results: At 24 months, the presence of low PTH levels increased by $26.7 \%$. The main independent factors for
\end{abstract}

low PTH levels were a low BMI, hemoglobin, and serum albumin and high serum calcium $(p<0.05)$. A persistently low PTH level at 24 months was associated with a decrease in the left ventricular ejection fraction (LVEF) and increase in valvular calcification $(p<0.05)$. Additionally, a decrease in PTH levels from normal or high to low values was associated with a decrease in LVEF and cardiac output (CO) and an increase in valvular calcification $(p<0.05)$. Furthermore, compared with those of the persistently low PTH level group, LVEF values were lower at 24 months in the group with a decrease from high/normal to low PTH level $(p<0.05)$. Conclusion: Persistently low PTH levels and changes in the PTH level from high/ normal to low were associated with cardiac function decline in MHD patients. Moreover, a PTH level change from high/ normal to low showed a stronger correlation with cardiac function decline.

(c) 2021 The Author(s)

Published by S. Karger AG, Basel karger@karger.com www.karger.com/mpp

Karger $\stackrel{\text { ' }}{5}$

BOPEN ACCESS
(C) 2021 The Author(s)

Published by S. Karger AG, Basel

This is an Open Access article licensed under the Creative Commons Attribution-NonCommercial-4.0 International License (CC BY-NC) (http://www.karger.com/Services/OpenAccessLicense), applicable to the online version of the article only. Usage and distribution for commercial purposes requires written permission. 


\section{Introduction}

Hemodialysis is the most common renal replacement therapy used to treat patients with ESRD. Although hemodialysis can be lifesaving for these patients, the mortality rate associated with dialysis is approximately $5-27 \%$ annually in developed countries [1]. Cardiovascular diseases (CVD) are the main causes of death in patients undergoing maintenance hemodialysis (MHD) [2]. In addition to traditional cardiovascular risk factors, chronic kidney disease-mineral and bone metabolism disorder has been shown to contribute to alterations in arterial structure and function [3]. This disorder has become a focus of research in recent years due to its association with the fracture risk, CVD, and mortality [4].

High parathyroid hormone (PTH) levels have been associated with cardiovascular calcification and mortality [5]. However, recent studies have also shown that a high proportion of patients receiving dialysis therapy have relatively low PTH levels. In addition, cardiovascular calcification is more prevalent in MHD patients who have low PTH levels than in those with normal or moderately elevated levels. And, this may be associated with low turn- over or adynamic bone disease [6]. Regardless, no studies have investigated the relationship between variability in low PTH levels and cardiac function in MHD patients.

The objective of this study is to analyze the variability in low PTH levels and cardiac function indices and to assess whether this variability is associated with changes in cardiac function. Furthermore, factors involved in the decreased PTH levels or cardiac function in these patients are identified.

\section{Materials and Methods}

\section{Study Population}

The study was designed as a prospective observational study. All patients included were selected from the Department of Hemodialysis Treatment Center of Zhong Nan Hospital of Wuhan University, China. The exclusion criteria were as follows: (1) dialysis duration $<3$ months; (2) primary parathyroid disease or receiving parathyroid surgery; (3) acute infection; (4) a history of serious CVD such as myocardial infarction, congestive heart failure, and malignant arrhythmia; (5) a family history of mental illness or a clear diagnosis with a mental illness; and (6) cerebrovascular event in previous 3 months. The patients underwent hemodialysis therapy twice or thrice a week using a standard dialysate $(1.5 \mathrm{mmol} / \mathrm{L}$ calcium). We included 231 patients with available serum PTH data

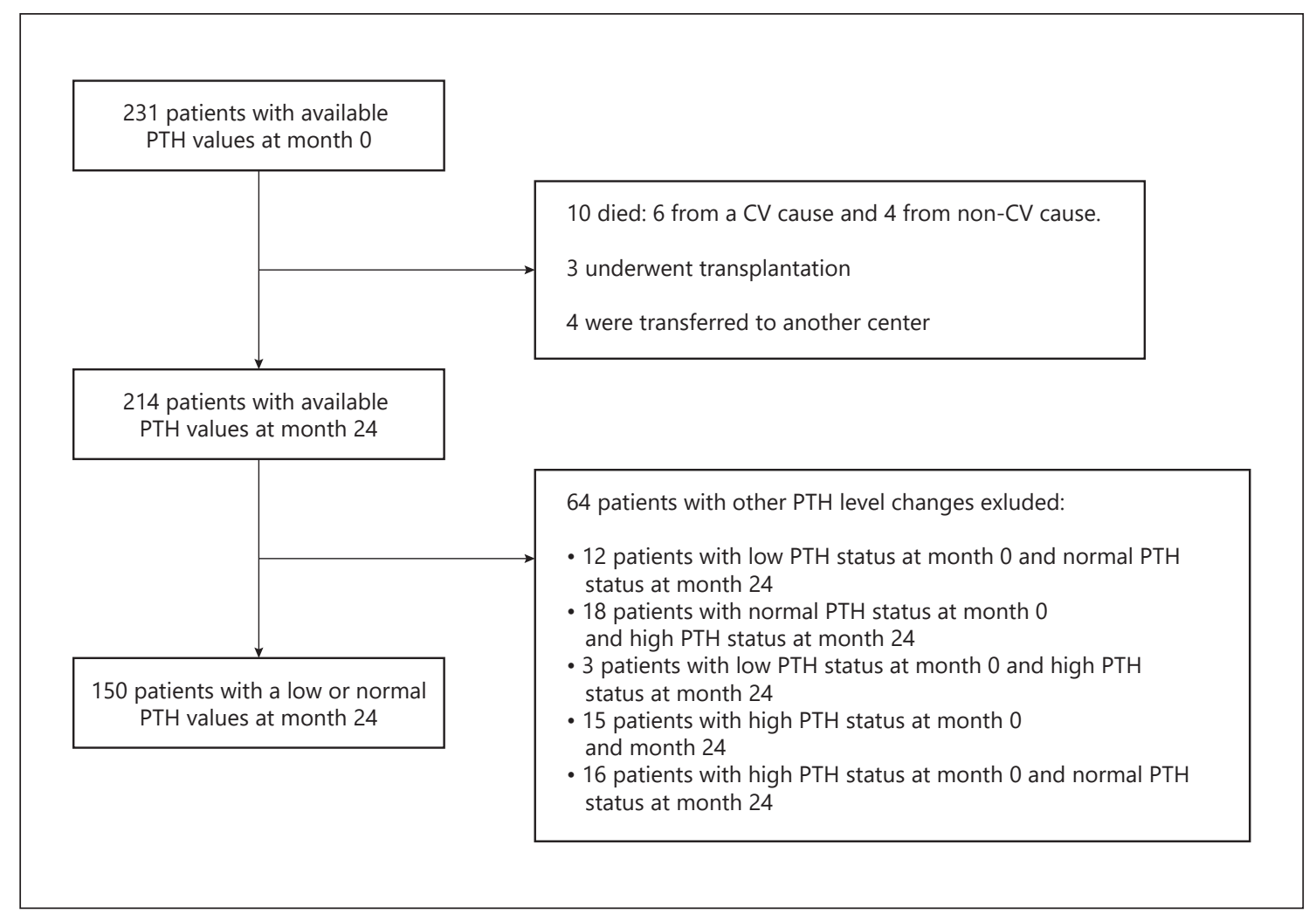

Fig. 1. Flow diagram of the study selection.

Low PTH and Cardiac Function Decline in MHD Patients 
Table 1. Baseline characteristics of the patients

\begin{tabular}{|c|c|c|c|c|}
\hline & Low-low group & High/normal-low group & Normal-normal group & $p$ value \\
\hline Patients, $n$ & 74 & 40 & 36 & - \\
\hline Age, years & $61.36 \pm 14.02$ & $60.58 \pm 13.83$ & $60.96 \pm 11.87$ & 0.334 \\
\hline Gender female, $n(\%)$ & $35(47.3)$ & $18(45.0)$ & $15(41.7)$ & - \\
\hline $\mathrm{BMI}, \mathrm{kg} / \mathrm{m}^{2}$ & $21.14 \pm 3.10$ & $20.26 \pm 3.43$ & $21.44 \pm 3.04$ & 0.582 \\
\hline Diabetes mellitus, \% & 42.5 & 42.9 & 43.1 & - \\
\hline Hypertension, \% & 33.5 & 31.9 & 34.7 & - \\
\hline \multicolumn{5}{|l|}{ Treatment of medication, $n(\%)$} \\
\hline Calcium-based P binders & $24(32.4)$ & $14(35.0)$ & $12(36.6)$ & 0.570 \\
\hline Sevelamer & $3(4.0)$ & $3(7.5)$ & $2(5.6)$ & - \\
\hline Lanthanum & $5(6.8)$ & $2(5.0)$ & $0(0.0)$ & - \\
\hline Calcitriol & $53(71.6)$ & $18(45.0)$ & $11(30.6)$ & 0.090 \\
\hline Alfacalcidol & $1(1.4)$ & $0(0.0)$ & $0(0.0)$ & - \\
\hline Red blood cell count, $\times 10^{9} / \mathrm{L}$ & $3.05 \pm 0.69$ & $3.89 \pm 1.74$ & $3.43 \pm 0.73$ & 0.000 \\
\hline Hemoglobin, g/L & $94.53 \pm 23.91$ & $99.99 \pm 21.97$ & $96.27 \pm 24.65$ & 0.013 \\
\hline Hematocrit, $\%$ & $28.83 \pm 7.13$ & $30.78 \pm 6.44$ & $32.90 \pm 7.09$ & 0.000 \\
\hline Serum albumin, g/L & $35.71 \pm 6.74$ & $36.65 \pm 7.53$ & $33.91 \pm 4.32$ & 0.139 \\
\hline Serum hs-CRP, mg/dL & $0.19(0.05-0.77)$ & $0.21(0.07-0.81)$ & $0.18(0.04-0.85)$ & 0.357 \\
\hline Serum creatinine, $\mu \mathrm{mol} / \mathrm{L}$ & $630.70 \pm 253.76$ & $710.69 \pm 297.07$ & $845.43 \pm 337.02$ & 0.319 \\
\hline Serum urea nitrogen, $\mu \mathrm{mol} / \mathrm{L}$ & $19.06 \pm 8.31$ & $21.23 \pm 9.79$ & $24.83 \pm 8.26$ & 0.903 \\
\hline Uric acid, $\mu \mathrm{mol} / \mathrm{L}$ & $381.32 \pm 149.68$ & $388.30 \pm 131.19$ & $355.28 \pm 75.46$ & 0.621 \\
\hline Serum calcium, mmol/L & $1.93 \pm 0.24$ & $1.98 \pm 0.20$ & $1.92 \pm 0.19$ & 0.588 \\
\hline Serum phosphorus, $\mathrm{mmol} / \mathrm{L}$ & $1.54 \pm 0.50$ & $1.81 \pm 0.67$ & $1.60 \pm 0.52$ & 0.375 \\
\hline \multicolumn{5}{|l|}{ Cardiac function index } \\
\hline LVEF, \% & $63.68 \pm 11.25$ & $64.60 \pm 12.77$ & $61.70 \pm 5.49$ & 0.260 \\
\hline
\end{tabular}

Continuous variables are shown as the mean and SD for normally distributed data and as the median and first quartile for nonnormally distributed data. PTH, parathyroid hormone; LVEF, left ventricular ejection fraction; CO, cardiac output.

on June 1, 2014 (month 0 ), and 214 patients remained in the study on June 30, 2016 (month 24). For the present study, we included patients with a low, normal, or high PTH status at month 0 and a low or normal PTH status at month 24 using serum PTH concentrations of $150-300 \mathrm{pg} / \mathrm{mL}$ as the reference range. Patients with other PTH status changes from month 0 to month 24 or whose PTH level information was unavailable were excluded $(n=64)$. Ultimately, 150 patients were included in the final analysis at baseline and were prospectively followed for 24 months (Fig. 1).

\section{Data Collection}

Data were collected using an electronic case-report system. The investigators were not aware of a current study of the PTH status and cardiac function. Baseline demographic, clinical, laboratory, and other general data were collected. Echocardiography was performed by a single experienced sonographer blinded to the clinical details of patients. All echocardiographic data were measured according to the guidelines of the American Society of Echocardiography (ASE) using the Sonoline G50 2-dimensional color Doppler ultrasound diagnostic instrument (Siemens Medical Solutions USA, Inc., Malvern, PA, USA). The left ventricular ejection fraction (LVEF) was measured using Simpson's method. The Dopplerestimated cardiac output was derived from systolic volume using the time-velocity integral of the flow through the left ventricular outflow tract (LVOT), the LVOT diameter, and heart rate during the imaging study. Cardiac output was calculated using the following formula: cardiac output $=$ heart rate (beats per minute $) \times$ timevelocity integral $(\mathrm{cm}) \times 3.1416 \times(\text { LVOT diameter }[\mathrm{cm}] / 2)^{2}\left(\mathrm{~cm}^{2}\right)$ $=$ milliliter per minute [7]. Valvular calcification was defined as the presence of one or more rings in the aortic or mitral valve [8]. The serum PTH concentrations were measured via second-generation chemiluminescent immunoassay PTH assay (Beckman Coulter, Inc.). The low-low group included patients with $\mathrm{PTH}<150 \mathrm{pg} / \mathrm{mL}$ at both months 0 and 24, the high/normal-low group included patients with PTH $\geq 150 \mathrm{pg} / \mathrm{mL}$ at month 0 , followed by a decrease to $\mathrm{PTH}<150 \mathrm{pg} / \mathrm{mL}$ at month 24 , and the normal-normal group included patients with PTH between 150 and $300 \mathrm{pg} / \mathrm{mL}$ at months 0 and 24 . All laboratory evaluations were performed locally. 
Table 2. Variables associated with a decrease in the PTH level

\begin{tabular}{llll}
\hline & \multicolumn{2}{l}{ High/normal-low } & \\
\cline { 2 - 4 } & OR & $95 \%$ CI & p value \\
\hline BMI $\left(\mathrm{kg} / \mathrm{m}^{2}\right)$ & 0.905 & $0.834-0.982$ & 0.017 \\
Hemoglobin $(\mathrm{g} / \mathrm{L})$ & 0.950 & $0.910-0.991$ & 0.019 \\
Serum albumin $(\mathrm{g} / \mathrm{L})$ & 0.952 & $0.909-0.997$ & 0.038 \\
Serum calcium $(\mathrm{mmol} / \mathrm{L})$ & 1.032 & $1.002-1.076$ & 0.001 \\
Treatment of medication & 1.130 & $0.991-1.271$ & 0.075 \\
$\quad$ Calcium-based P binders & - & - & - \\
$\quad$ Sevelamer & - & - & - \\
$\quad$ Lanthanum & 1.020 & $0.909-1.032$ & 0.084 \\
$\quad$ Calcitriol & - & - & - \\
$\quad$ Alfacalcidol & & \multicolumn{2}{l}{} \\
\hline
\end{tabular}

The adjusted OR was obtained from the multivariate logistic regression model. Estimates are reported for all independent predictors included in the final models. CI, confidence interval; OR, odds ratio; $\mathrm{PTH}$, parathyroid hormone.
Statistical Analysis

All statistical analyses were performed with the Statistical Product and Service Solutions software (SPSS, version 22.0; Chicago, IL, USA). Normally distributed variables are presented as the mean \pm standard deviation with $95 \%$ confidence interval. We used 2 -sample $t$ tests or the 1-way ANOVA test to compare continuous variables across groups and Pearson's $\chi^{2}$ test $\left(\chi^{2}\right)$ to compare categorical variables across groups. We used multivariate logistic regression analysis to identify predictive factors of cardiac function changes and PTH status variability between months 0 and 24 and determined the odds ratios for each factor. The analysis was adjusted for potential confounders. For all analyses, a 2 -sided $p$ value $<0.05$ was considered statistically significant.

\section{Results}

\section{Baseline Characteristics}

The baseline characteristics of both cohorts are shown in Table 1. Among the 150 patients, 74 patients (49.3\%) had a low PTH status from months 0 to 24 (low-low group), 36 patients $(24 \%)$ had a normal PTH status at months 0 and 24 (normal-normal group), and 40 patients (26.7\%) had a high or normal PTH status at month 0 that decreased to a low PTH status at month 24 (high/normallow group). No significant differences were found in age, sex ratio, BMI, prevalence of diabetes and hypertension, treatment of phosphate binder or vitamin $\mathrm{D}$, blood pressure, and dialysis duration among the 3 groups.

\section{Independent Risk Factors for a Decrease in the PTH Level}

The prevalence of low PTH levels was $49.3 \%$ at baseline and $76.0 \%$ after 24 months. To identify factors poten- tially associated with changes in the PTH levels from months 0 to 24 , we performed multivariate logistic regression analysis in the high/normal-low group. Decreases in BMI, hemoglobin, and serum albumin were associated with the risk of a shift from a "high/normal" to a "low" status ( $p=0.017,0.019$, and 0.038 , respectively). The decrease in the PTH level from months 0 to 24 was also associated with an increase in serum calcium $(p=$ 0.001) (Table 2).

\section{Independent Risk Factors for a Decrease in Cardiac Function}

Cardiac function indices, such as the LVEF, CO, and valvular calcification, were assessed in the 3 subgroups at 0 and 24 months using multivariate logistic regression analysis (Table 3). Low-low PTH variability resulted in a decrease in $\mathrm{CO}(p=0.034)$ and an increase in valvular calcification $(p=0.007)$. In contrast, high/normal-low PTH variability increased the risk of a decline in cardiac function ( $p=0.014$ for LVEF; $p<0.001$ for CO; $p<0.001$ for valvular calcification). In addition to age ( $p<0.001$ for LVEF; $p<0.001$ for CO; $p<0.001$ for valvular calcification), a high serum calcium level was strongly associated with a decreased $\operatorname{LVEF}(p=0.013)$ and increased valvular calcification $(p=0.003)$. A decrease in hemoglobin was associated with reduced $\mathrm{CO}(p=0.036)$.

\section{Correlations between PTH Level Changes and Cardiac Function Decline}

We also analyzed changes in the cardiac function indices among the 3 groups (Table 4). Low-low PTH variability was associated with a decrease in the LVEF $(p=$ 
Table 3. Variables associated with a decline in cardiac function

\begin{tabular}{|c|c|c|c|c|c|c|}
\hline \multirow[t]{2}{*}{ Index } & \multicolumn{2}{|l|}{ LVEF } & \multicolumn{2}{|l|}{$\mathrm{CO}$} & \multicolumn{2}{|c|}{ Valvular calcification } \\
\hline & OR $(95 \% \mathrm{CI})$ & $p$ value & OR (95\% CI) & $p$ value & OR (95\% CI) & $p$ value \\
\hline Age (years) & $1.41(1.13-1.55)$ & $<0.001$ & $1.43(1.12-1.57)$ & $<0.001$ & $1.41(1.12-1.46)$ & $<0.001$ \\
\hline Low-low PTH & $1.98(0.78-5.05)$ & 0.154 & $2.74(1.08-6.95)$ & 0.034 & $1.91(1.47-3.02)$ & 0.007 \\
\hline High/normal-low PTH & $5.10(1.38-8.76)$ & 0.014 & $2.85(2.29-8.21)$ & $<0.001$ & $1.94(1.34-2.63)$ & $<0.001$ \\
\hline Hemoglobin $(\mathrm{g} / \mathrm{L})$ & $0.48(0.16-1.41)$ & 0.052 & $0.16(0.03-0.88)$ & 0.036 & $1.82(0.46-7.09)$ & 0.387 \\
\hline Serum calcium $(\mathrm{mmol} / \mathrm{L})$ & $3.19(1.28-7.97)$ & 0.013 & $0.66(0.29-1.48)$ & 0.314 & $2.53(1.21-5.31)$ & 0.003 \\
\hline
\end{tabular}

Adjusted ORs were obtained from the multivariate logistic regression model. Estimates are reported for all independent predictors included in the final models. CI, confidence interval; OR, odds ratio; PTH, parathyroid hormone; LVEF, left ventricular ejection fraction; $\mathrm{CO}$, cardiac output.

Table 4. Correlation between changes in $\mathrm{PTH}$ and a decline in cardiac function

\begin{tabular}{|c|c|c|c|c|c|c|c|c|c|}
\hline \multirow[t]{2}{*}{ Cardiac function index } & \multicolumn{3}{|c|}{ Low-low group } & \multicolumn{3}{|c|}{ High/normal-low group } & \multicolumn{3}{|c|}{ Normal-normal group } \\
\hline & month 0 & month 24 & $p$ value & month 0 & month 24 & $p$ value & month 0 & month 24 & $p$ value \\
\hline LVEF, \% & $63.68 \pm 11.2$ & $60.51 \pm 10.39$ & 0.045 & $64.60 \pm 12.77$ & $59.50 \pm 13.03$ & 0.023 & $61.70 \pm 5.49$ & $62.11 \pm 11.20$ & 0.887 \\
\hline $\mathrm{CO}, \mathrm{mL} / \mathrm{min}$ & $5.36 \pm 1.35$ & $5.23 \pm 1.24$ & 0.680 & $5.29 \pm 0.82$ & $4.35 \pm 0.98$ & 0.002 & $5.41 \pm 1.00$ & $5.83 \pm 1.20$ & 0.281 \\
\hline Calcification/noncalcification & $20 / 54$ & $38 / 36$ & 0.004 & $15 / 25$ & $24 / 16$ & 0.043 & $12 / 24$ & $15 / 21$ & 0.598 \\
\hline
\end{tabular}

Continuous variables are shown as the mean and SD for normally distributed data and as the median and first quartile for nonnormally distributed data. Adjusted ORs were obtained from the multivariate logistic regression model. Estimates are reported for all independent predictors included in the final models. PTH, parathyroid hormone; LVEF, left ventricular ejection fraction; CO, cardiac output.

$0.045)$ and an increase in valvular calcification $(p=0.004)$, whereas high/normal-low PTH variability increased the risk of a decline in cardiac function $(p=0.023$ for LVEF; $p=0.002$ for $\mathrm{CO} ; p<0.043$ for valvular calcification). We did not observe a statistical significant difference in the normal-normal group $(p>0.05)$. Furthermore, compared with those of the PTH low-low group, the LVEF value was lower in high/normal-low PTH variability ( $p=$ 0.045) (Table 5).

\section{Discussion}

The incidence of CVD is higher in patients with chronic kidney disease than in the general population [9]. And, chronic renal failure is often accompanied by left ventricular hypertrophy, left ventricular dilatation, myocardial dysfunction, and overt heart failure [10]. Although many previous studies have confirmed that PTH is an important independent risk factor in MHD patients with CVD, most of these studies have focused on the relationship between high PTH levels and CVD events [11]. A previous study which showed the presence of low $\mathrm{PTH}$ levels was 23.0\% in MHD patients (172/748) [12]. In patients after parathyroidectomy, persistent hypoparathyroidism occurred in $6(1.5 \%)$ of 402 patients [13]. Conversely, research on the relationship between low PTH levels and heart dysfunction is rare. In this study, low PTH levels in MHD patients were analyzed, and correlations between variability in low PTH levels and cardiac function were explored.

In this study, the incidence of low PTH was found to be increased in MHD patients after 24 months. A decrease from a normal/high to a low PTH level was related to decreases in the BMI, hemoglobin, and serum albumin as well as an increase in serum calcium. A previous study by Drechsler et al. [14] investigated the effect of low PTH levels on the mortality risk in MHD patients and showed that a decrease in PTH over 3 months was associated with a higher risk of all-cause mortality in those with a low BMI. In that study, the changes in BMI had a probable link to all-cause mortality through protein-energy wasting, malnutrition, or both. In our cohort, decreased PTH was also associated with a lower BMI and serum albumin. 
Table 5. The characteristics of the high/normal-low and low-low groups at month 24

\begin{tabular}{lclllll}
\hline Index & Low-low group & $\begin{array}{l}\text { High/ } \\
\text { normal-low group }\end{array}$ & T or $\chi^{2}$ & $p$ value $95 \%$ CI \\
\hline LVEF, \% & $60.51 \pm 10.39$ & $59.50 \pm 13.03$ & 1.899 & 0.045 & $(1.88,2.23)$ \\
CO, mL/min & $5.23 \pm 1.24$ & $4.35 \pm 0.98$ & 0.414 & 0.680 & $(-0.48$ to 1.73$)$ \\
Calcification/noncalcification & $38 / 36$ & $24 / 16$ & 2.186 & 0.124 & - \\
\hline
\end{tabular}

Continuous variables are shown as the mean and SD for normally distributed data and as the median and first quartile for nonnormally distributed data. LVEF, left ventricular ejection fraction; CO, cardiac output.

Wasting is common in ESRD and represents the following processes: a severe and complex process of metabolic acidosis-induced intestinal dysbiosis; systemic inflammation with activation of complements, endothelin-1, and the renin-angiotensin-aldosterone axis; anabolic hormone resistance; energy expenditure elevation; and uremic toxin accumulation [15].

Additionally, we found that high serum calcium was another strong risk factor for decreases in PTH from high or normal to low levels. The feedback effect of hypercalcemia on parathyroid tissue inhibits PTH synthesis and secretion. Moreover, although our study showed no significant relationship between the PTH and serum phosphorus levels, this analysis could have been affected by various factors, such as the sample size, clinical features, and treatment status. Thus, more in-depth multicenter clinical research with a large sample needs to be conducted for confirmation. Multiple logistic regression analysis also revealed an association of high serum calcium with LVEF and valvular calcification. When PTH is excessively reduced, serum calcium absorption also decreases, and more exogenous calcium is absorbed. Hypercalcemia leads to excess calcium deposition in soft tissues and vessels, which significantly increases the risk of low bone turnover, bone fracture, and coronary artery calcification [16].

The 3 groups of patients in our study all developed anemia, and the condition in the high/normal-low group patients had worsened after 24 months. Specifically, hemoglobin, red blood cell count, and hematocrit decreased significantly. PTH is an important regulator of bone metabolism. MHD is often accompanied by low-turnover osteopathy when serum PTH is excessively inhibited, resulting in changes in the bone marrow hematopoietic microenvironment. Thus, hematopoietic stem cell activation is limited, and erythrocyte production is reduced. Hepcidin, which has an important function in iron levels and anemia, may be another important and related factor.
Previous studies have reported that hepcidin is involved in metabolism of bone minerals [17]. Nonetheless, the data from this study are insufficient to elucidate the specific mechanism underlying the relationship between low PTH variability and anemia.

Cardiac function decline was paralleled by a decrease in hemoglobin in MHD patients. Recent increasing evidence has shown that anemia is an independent risk factor for CVD [18]. Long-term anemia can alter hemodynamics and lead to a compensatory increase in cardiac output, left ventricular hypertrophy, left ventricular dilation, and growth [19].

In this study, continuously low PTH levels and a decrease from a high or normal to a low PTH status were strong risk factors for cardiac function decline. Moreover, the cardiac function decline was more obvious in the high/normal-low group, which was in agreement with a previous study by Naves-Diaz et al. [20] in which low PTH levels in dialysis patients were associated with a risk of cardiovascular morbidity. Furthermore, this result supports the findings of a previous report showing that an excessively reduced PTH level induces low bone turnover disease and arterial calcification [21]. Considering the mechanisms underlying the association between high/normal-low PTH and cardiac function, we hypothesize that the PTH-related peptide (PTHrP)/PTH receptor regulation system plays an important role in the specific regulatory processes of myocardial cells and is associated with cardiac hypertrophy and heart failure. The PTH receptor, which is a G protein-coupled receptor consisting of 7 transmembrane segments, is present on myocardial cells, and binding of PTH or PTHrP to the receptor stimulates normal myocardial cell function [22]. When the serum PTH levels decrease, receptor binding and stimulation also decrease, resulting in insufficient myocardial cell function and, ultimately, cardiac function decline. Nonetheless, the reason why a progressive decrease in $\mathrm{PTH}$, but not 
sustained low PTH levels, is more related with the changes in cardiac function remains a matter of conjecture.

\section{Conclusion}

The findings of this study suggest that variability in a low PTH serum status is a strong risk factor for cardiac function decline and should be given attention in clinical research.

\section{Statement of Ethics}

This study was approved by the Medical Ethics Committee of Zhong Nan Hospital of Wuhan University (2016019), and informed consent was obtained from all patients.

\section{Conflict of Interest Statement}

The authors declare no conflicts of interest.

\section{Funding Sources}

This article has no funding sources.

\section{Author Contributions}

Xiao-han Lu and co-first author Meng-si Li contributed to the design of the study, data analysis, and writing and editing of the manuscript. Yao-yao Li and Yan-dan Zheng performed article review. Gao Ping and Xiao-yan Wu assisted in modifying the manuscript.

\section{References}

1 Ortiz A, Covic A, Fliser D, Fouque D, Goldsmith D, Kanbay M, et al. Epidemiology, contributors to, and clinical trials of mortality risk in chronic kidney failure. Lancet. 2014; 383(9931):1831-43.

2 Drew DA, Weiner DE, Tighiouart H, Scott T, Lou K, Kantor A, et al. Cognitive function and all-cause mortality in maintenance hemodialysis patients. Am J Kidney Dis. 2015;65(2):30311.

3 Merle E, Roth H, London GM, Jean G, Hannedouche T, Bouchet JL, et al. Low parathyroid hormone status induced by high dialysate calcium is an independent risk factor for cardiovascular death in hemodialysis patients. Kidney Int. 2016;89(3):666-74.

4 Seifert ME, Hruska KA. The Kidney-vascularbone axis in the chronic kidney disease-mineral bone disorder. Transplantation. 2016;100(3): 497-505.

5 Natoli JL, Boer R Nathanson BH, Ross MM, Chiroli S, Goodmanan WG, et al. Is there an association between elevated or low serum levels of phosphorus, parathyroid hormone, and calcium and mortality in patients with end stage renal disease? A meta-analysis. Bmc Nephrol. 2013;14:88.

6 Fouque D, Roth H, Pelletier S, London GM, Hannedouche T, Jean G, et al. Control of mineral metabolism and bone disease in haemodialysis patients: which optimal targets? Nephrol Dial Transplant. 2013;28(2):360-7.

7 Lang RM, Bierig M, Devereux RB, Flachskampf FA, Foster E, Pellikka PA, et al. Recommendations for chamber quantification: a report from the American Society of Echocardiography's Guidelines and Standards Committee and the Chamber Quantification Writing Group, developed in conjunction with the European As- sociation of Echocardiography, a branch of the European Society of Cardiology. J Am Soc Echocardiogr. 2005;18(12):1440-63.

8 Li M, Ye ZC, Li CM, Zhao WB, Tang H, Liu X, et al. The influence of cardiac valvular calcification on all-cause and cardiovascular mortality in maintenance hemodialysis patients. Int Urol Nephrol. 2020;52(5):943-51.

$9 \mathrm{Pu} \mathrm{L}$, Zou Y, Wu SK, Wang F, Zhang Y, Li GS, et al. Prevalence and associated factors of depressive symptoms among chronic kidney disease patients in China: results from the Chinese Cohort Study of Chronic Kidney Disease (CSTRIDE). J Psychosom Res. 2020;128:109869.

10 Thompson S, James M, Wiebe N, Hemmelgarn B, Manns B, Klarenbach S, et al. Cause of death in patients with reduced kidney function. J Am Soc Nephrol. 2015;26(10):2504-11.

11 Bansal N, Zelnick L, Robinson-Cohen C, Hoofnagle AN, Ix JH, Lima JA, et al. Serum parathyroid hormone and 25-hydroxyvitamin D concentrations and risk of incident heart failure: the Multi-Ethnic Study of Atherosclerosis. J Am Heart Assoc. 2014;3(6):e001278.

12 Dukkipati R, Kovesdy CP, Colman S, Budoff MJ, Nissenson AR, Sprague SM, et al. Association of relatively low serum parathyroid hormone with malnutrition-inflammation complex and survival in maintenance hemodialysis patients. J Ren Nutr. 2010;20(4):243-54.

13 Hermann M, Ott J, Promberger R, Kober F, Karik M, Freissmuth M. Kinetics of serum parathyroid hormone during and after thyroid surgery. Br J Surg. 2008;95(12):1480-7.

14 Drechsler C, Grootendorst DC, Boeschoten EW, Krediet RT, Wanner C, Dekker FW, et al. Changes in parathyroid hormone, body mass index and the association with mortality in dialysis patients. Nephrol Dial Transplant. 2011; 26(4):1340-6.
15 Zha Y, Qian Q. Protein nutrition and malnutrition in CKD and ESRD. Nutrients. 2017;9(3): 208.

16 Jean GLD, Genet L, Legrand E, Kuentz F, Moreau-Gaudry X, Fouque D, et al. Association between very low PTH levels and poor survival rates in haemodialysis patients: results from the French ARNOS cohort. Nephron Clin Pract. 2011;118(2):C211-6.

17 Del Vecchio L, Locatelli F. New treatment approaches in chronic kidney disease-associated anaemia. Expert Opin Biol Ther. 2014;14(5): 687-96.

18 Leaf DE, Goldfarb DS. Interpretation and review of health-related quality of life data in CKD patients receiving treatment for anemia. Kidney Int. 2009;75(1):15-24.

19 Chang JM, Chen SC, Huang JC, Su HM, Chen HC. Anemia and left ventricular hypertrophy with renal function decline and cardiovascular events in chronic kidney disease. Am J Med Sci. 2014;347(3):183-9.

20 Naves-Diaz M, Passlick-Deetjen J, Guinsburg A, Marelli C, Fernandez-Martin JL, RodriguezPuyol D, et al. Calcium, phosphorus, PTH and death rates in a large sample of dialysis patients from Latin America. The CORES Study. Nephrol Dial Transplant. 2011;26(6):1938-47.

21 Hernandes FR, Canziani ME, Barreto FC, Santos RO, Moreira VM, Rochitte CE, et al. The shift from high to low turnover bone disease after parathyroidectomy is associated with the progression of vascular calcification in hemodialysis patients: a 12-month follow-up study. PLoS One. 2017;12(4):e0174811.

22 Halapas A, Diamanti-Kandarakis E, Kremastinos D, Koutsilieris M. The PTHrP/PTH.1-R bioregulation system in cardiac hypertrophy: possible therapeutic implications. In Vivo. 2006;20(6B):837-44. 